\title{
INTERACTIONS AMONG SPATIAL FREQUENCY AND ORIENTATION CHANNELS ADAPTED CONCURRENTLY
}

\author{
Mark W. Greenlee and Svein Magnussen \\ Neurologische Universitätsklinik mit Abteilung für Neurophysiologie, Hansastr. 9, 7800 Freiburg, F.R.G. \\ and Institute of Psychology, University of Oslo, Box 1094, N-0317 Oslo 3, Norway
}

(Received 9 February 1988; in revised form I June 1988)

\begin{abstract}
Interactions between size and orientation-specific mechanisms in the human visual system were investigated using a sequential adaptation technique. Subjects adapted to a vertical, $4 \mathrm{c} / \mathrm{deg}$ high-contrast (0.7) sinewave grating that was interleaved at a rate of $0.5 \mathrm{~Hz}$ with another adapting grating differing either in (1) spatial frequency or (2) orientation. Before and after adaptation contrast thresholds were measured for a vertical $4 \mathrm{c} / \mathrm{deg}$ sinewave test grating. The resultant elevation in contrast threshold was plotted as a function of the (1) spatial frequency or (2) orientation differences between the first and second adapting gratings. Maximum threshold elevation was found when both adapting gratings shared the same spatial frequency and orientation. Minimum elevations were found when the second grating's spatial frequency or orientation differed by approx. 1.5 octaves or $45 \mathrm{deg}$, respectively. Beyond these values threshold elevations reapproached the baseline value measured in a control condition, where the $4.0 \mathrm{c} / \mathrm{deg}$ adapting grating was interleaved with a blank. The minimum threshold elevations were $0.2-0.3 \mathrm{log}$ units below the baseline level. The results suggest the existence of inhibitory interactions between neural mechanisms tuned to the size and orientation of retinal images.
\end{abstract}

Spatial vision Adaptation Channels Lateral inhibition Spatial frequency Orientation

\section{INTRODUCTION}

Several lines of evidence converge to support the theory that the visual system of the cat, monkey and man contains spatially localized neural mechanisms, or "channels", responding only to a restricted range of sizes (spatial frequencies) and orientations (Braddick et al., 1978; Rose and Dobson, 1985; Shapley and Lennie, 1985). The most convincing psychophysical evidence for such channels come from selective adaptation experiments, wherein the observer views a high-contrast grating of a particular spatial frequency and orientation and the pre- and post-adaptation contrast thresholds are determined for test gratings of various spatial frequencies and orientations. The range of test stimuli over which a threshold elevation is observed is thought to reflect the bandwidth of the underlying neural mechanism for the stimulus parameter under investigation (Blakemore and Campbell, 1969; Blakemore and Nachmias, 1971; Georgeson and Harris, 1984). Experiments using complex gratings containing two or more spatial frequencies or orientations indicate that composite adapting stimuli may induce less contrast threshold elevation at either component frequency/orientation than when these components are adapted to alone (Tolhurst, 1972; Nachmias et al., 1973; Stecher et al., 1973; Tolhurst and Barfield, 1978; Georgeson, 1980). These results led to the suggestion that the neural channels underlying this stimulus selectivity are not completely independent, but that each channel receives inhibitory input from channels coding neighbouring spatial frequencies and orientations. Further psychophysical evidence for this type of spatial frequency and orientation-specific lateral inhibition comes from analogous studies on the suprathreshold tilt aftereffect (Magnussen and Kurtenbach, 1980; Kurtenbach and Magnussen, 1981) and from simultaneous contrast experiments studying the effects of spatial frequency (Klein et al., 1974) and orientation (Carpenter and Blakemore, 1973; Kurtenbach and Magnussen, 1981).

However, the interpretation of the composite adaptation experiments in terms of interchannel inhibition assumes that the processing of stimulus components is independent of the context in which they are embedded. Such a 
view is complicated by several factors. For example, the weak effect of a squarewave adapting grating on the third harmonic frequency (Tolhurst, 1972; Nachmias et al., 1973) could be due to the fact that the edges of the squarewave grating may be less effective in adapting mechanisms which would normally respond to one or more harmonic frequencies of the squarewave (Greenlee and Magnussen, 1987. 1988; von der Heydt et al., 1986; von der Heydt, 1987). Klein and Stromeyer (1980) have shown that the first and third harmonics of a squarewave can be effective adaptors, if they are "separately visible" during adaptation. Swift and Smith (1985) have argued that weak adaptation effects following composite adaptation could be caused by the local contrast beats occurring in the physical luminance profile of complex gratings whenever the component frequencies approach a similar value. These experiments therefore suggest that complex adapting stimuli in which two or more sinewaves are superimposed may not be adequate to test the inter-channel interaction hypothesis.

We here present new evidence for inhibitory interactions between neural mechanisms based on the technique of sequential adaptation (Greenlee and Magnussen, 1987). This technique permits concurrent adaptation to two spatial frequencies or orientations in the same retinal region and separates genuine interactions between mechanisms from the confounding effects of stimulus complexity. The experimental rationale is simple: to build up an aftereffect, adaptation need not be continuous but can be interrupted for short periods of time without reducing the size of the resultant aftereffect, provided total adaptation time is kept constant (Magnussen and Greenlee, 1986). We take advantage of this feature of adaptation by presenting two adapting gratings alternating in time. These gratings would adapt the same or different mechanisms depending on their similarity on the stimulus parameter in question, e.g. spatial frequency or orientation. It follows that the degree to which one adapting grating affects the elevation in contrast threshold caused by the other adapting grating reflects the bandwidth of the adapted mechanism. More importantly, this method can be used to measure the extent to which that mechanism can be affected by adaptation occurring outside of the channel's own operating range, and thus can reveal interactions between neighbouring channels.

\section{METHOD}

Sinewave gratings were produced on a highresolution cathode ray tube (Joyce Electronics, U.K.) under microprocessor control. The screen was masked off to a circular field of $11 \mathrm{deg}$ visual angle, which was viewed at a distance of $114 \mathrm{~cm}$. Viewing distance and head orientation was held constant using a chin forehead rest. The mean luminance of the screen was $150 \mathrm{~cd} / \mathrm{m}^{2}$. The linearity of the voltage contrast function was measured and controlled for on a regular basis using a spot photometer. Contrast thresholds were measured using a twoalternative forced-choice method where the test grating was presented randomly for $1 \mathrm{sec}$ in one of two $2.5 \mathrm{sec}$ temporal intervals. The brief pause before and after presentation of the test grating assured that the visual field was clear of any transient afterimages and that no form of forward and/or backward masking occurred. The intervals were delineated by auditory signals and the observer indicated in which interval he thought the grating appeared by pressing the appropriate button. According to the correctness of the observer's response. the computer then chose the next test contrast guided by a maximum-likelihood search algorithm (Lieberman and Pentland, 1982). All thresholds are based on 30 trials giving an average of 810 reversals of the contrast staircase. It has been shown that the reliability of this threshold search algorithm reaches an asymptote around 30 trials (Lieberman and Pentland, 1982). The data presented in Figs 2 and 4 are the means of at least two such measurements. The standard errors of the individual thresholds measurements usually do not exceed $1 \mathrm{~dB}$ in contrast.

The test gratings were vertically oriented with a constant spatial frequency of $4.0 \mathrm{c} / \mathrm{deg}$ and a temporal frequency of $1 \mathrm{~Hz}$. Of the two adapting gratings, one was fixed al $4.0 \mathrm{c} / \mathrm{deg}$ in the vertical orientation and the spatial frequency or orientation of the second adapting grating was varied in constant half-octave steps, while the adapting contrast was held constant at 0.7 . Contrast is defined as the ratio between the difference between the maximum and minimum luminance levels and their sum. The two adapting gratings were presented sequentially at a rate of $0.5 \mathrm{~Hz}$ over an $8 \mathrm{~min}$ adapting period. During adaptation the subject fixated along a small (1 deg) fixation circle and the spatial phase of the adapting grating was reversed at a temporal frequency of $1 \mathrm{~Hz}$. This procedure was done to 
avoid the induction of retinal afterimages and to create an adaptation effect that was independent of spatial phase. Post-adaptation thresholds were measured according to a test-readapt regime with $15 \mathrm{sec}$ readaptation periods interleaved with test trials.

To be able to compare the extent of interaction between the effects of the two adapting gratings, a control condition was conducted which measured the elevation in contrast threshold resulting when the standard $4 \mathrm{c} / \mathrm{deg}$ adapting grating was interleaved at the same rate with a blank field of same mean luminance. Since there is no spatial contrast in such a blank field, we were thus able, in this way, to measure the extent of adaptation in the mechanism tuned to the $4 \mathrm{c} / \mathrm{deg}$ test grating under the assumption that the only effect, if any, of the interleaving exposures to a blank field would be a partial decay of adaptation.

In the experiments on orientational effects, the screen was viewed monocularly through a two-channel optical system. In one channel the test and standard adapting grating were presented and in the other channel the orientation of the second adapting grating was varied in constant angular steps by changing the orientation of a Dove prism (see Fig. 1). Computer control of electronic shutters guaranteed proper synchronization of the test and adapting fields. Due to the optics, the mean luminance was reduced to $30 \mathrm{~cd} / \mathrm{m}^{2}$ at the eye. Four observers (MWG, SM, FH and WD) with normal or corrected-to-normal vision participated. All subjects had extensive training in similar experiments; WD was naive with respect to the aims of the study.

\section{RESULTS}

\section{Spatial-frequency interactions}

Figure 2 shows the results of the first experiment for the three observers tested, where the elevation in contrast threshold for the $4 \mathrm{c} / \mathrm{deg}$ grating is plotted as a function of the spatial frequency of the second (variable) adapting grating. The horizontal arrow and dashed line present the threshold elevation induced when the standard adapting grating was interleaved with a blank field of mean luminance.

The dual-grating adaptation function has a "sombrero" shape with a central range of spatial frequencies showing summation of adaptation effects between the two adapting gratings.

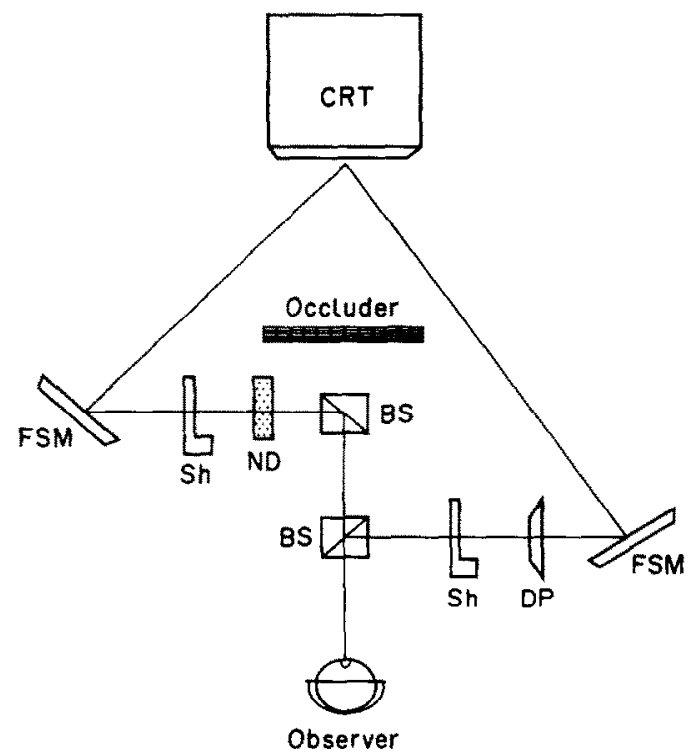

Fig. 1. $\Lambda$ schematic representation of the optical systcm developed to test the effect of orientation on threshold elevation. Abbreviations: $F S M=$ front surface mirror; $\mathrm{Sh}=$ shutter: $\mathrm{ND}=$ neutral density filter; $\quad \mathrm{BS}=$ beam splitter; $\mathrm{DP}=$ Dove prism.

Clearly, adapting gratings having a similar spatial frequency as that of the fixed adapting grating should stimulate the same mechanism centered at the test frequency of $4 \mathrm{c} / \mathrm{deg}$. For gratings of the same spatial frequency, the additional build-up of adaptation can be predicted based on the time course of adaptation to a single grating. Previous experiments indicate a difference between 0.2 and $0.4 \log$ units in threshold elevation resulting from 4 and $8 \mathrm{~min}$ of adaptation for different observers (Magnussen and Greenlee, 1985). Beyond this central summating range there is a second range where the elevation in threshold is reduced below the baseline level of the control condition, where the fixed adapting grating was interleaved with a blank field. This is followed by a third range, where the threshold elevation again approaches the baseline. There is a slight (approx. $0.1 \log$ unit) difference in threshold elevation obtained between the control condition and the condition where the two adapting gratings differed by 2 octaves or more for all three subjects tested. This finding suggests a slight global effect of the second adapting grating of the same orientation irrespective of spatial frequency. Correcting for this effect, the profiles show an approximate 2 octave summative region, surrounded by approximate 1 octave subtractive regions to either side. The full bandwidth of the central adapting region ( 1.2 octaves 


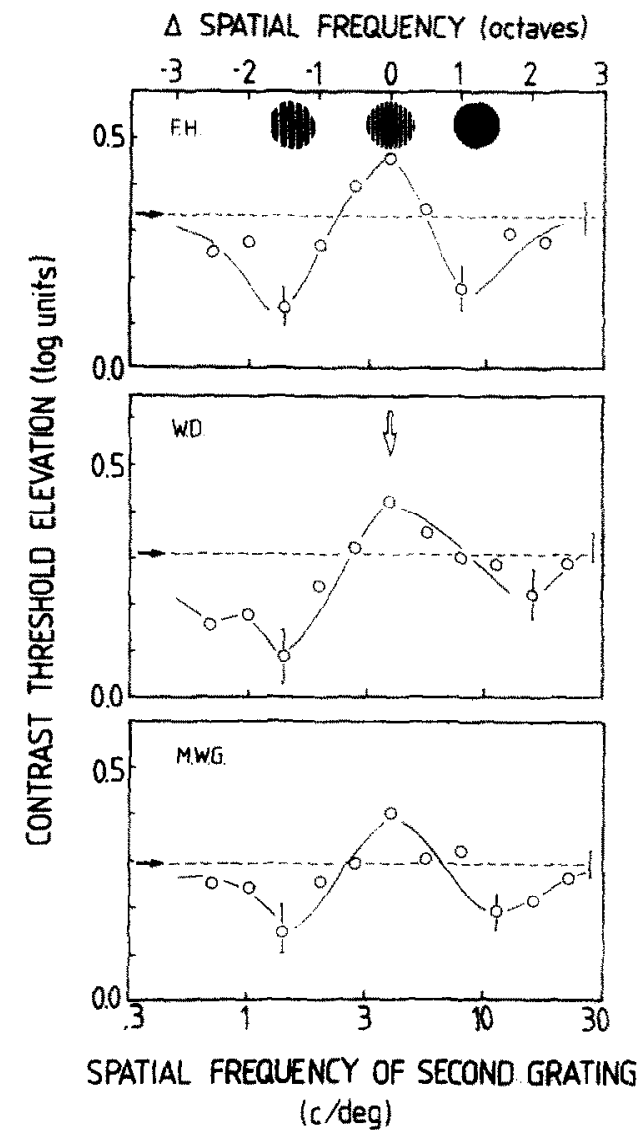

Fig. 2. The elevation in contrast threshold for detecting a $4 \mathrm{c} / \mathrm{deg}$ vertical grating following $8 \mathrm{~min}$ sequential adaptation to a high-contrast $4 \mathrm{c} / \mathrm{deg}$ vertical grating alternating with a second grating, as a function of the spatial frequency of the second grating. Dashed lines indicate the threshold elevation produced by adapting to a $4.0 \mathrm{c} / \mathrm{deg}$ grating alternating with a blank field. The data points are the mean values of, at least, two separate measurements and the error bars represent \pm 2 SEM. The grating insets (above) depict the spatial frequency of the second adapting grating when it was identical to the first grating (centre), or when it had its most pronounced subtractive effect on the resultant elevation in threshold (left and right). Results shown separately for subjects $F H, W D$ and $M W G$.

at half height) corresponds approximately to the tuning width of threshold elevation in single grating experiments (Blakemore and Campbell, 1969; Björklund and Magnussen, 1981; Georgeson and Harris, 1984).

To facilitate comparisons between subjects the findings shown in Fig. 2 have been normalized by assigning the maximum elevation in threshold the value of 1 for cach subject separately and plotting all remaining threshold elevations as a ratio of the maximum value. The results of this reanalysis are shown in Fig. 3 for all three observers. Normalized threshold elevation is now plotted as a function of the octave difference between the first and second

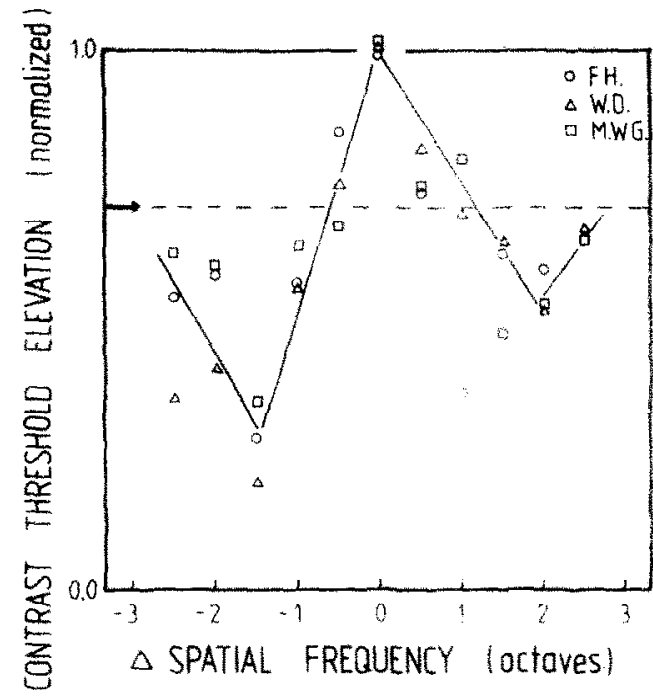

Fig. 3. The data in Fig. 2 are replotted after normalizing by assigning the value of 1 to the maximum threshold elevation and relating all other values to that maximum. Data for the three observers are shown. The straight lines have been fitted by eye.

adapting gratings. The straight lines have been fitted by eye and reveal that the reduction of threshold elevation is an asymmetric function of spatial frequency. For adapting frequencies above the $4 \mathrm{c} / \mathrm{deg}$ standard, the reduction in threshold elevation is less than for adapting gratings having a lower spatial frequency. Such an asymmetry may reflect differences in inhibitory interactions depending on the preferred spatial frequency of the underlying neural mechanisms.

\section{Orientation interactions}

We next investigated the effect of varying the orientation of the second adapting grating on the resultant elevation in contrast threshold. The results are shown for observer SM for two different adapting and test spatial frequencies: $4 \mathrm{c} / \mathrm{deg}$ (upper panel) and $8 \mathrm{c} / \mathrm{deg}$ (lower panel). Contrast threshold elevation (in log units) is plotted as a function of the orientation difference between the first and second adapting grating. A maximum threshold elevation occurs when both gratings have the same orientation as the test grating (vertical). For both spatial frequencies tested, minima occurred when the first and second adapting gratings differed by 45 angular deg. As has been reported earlier (Movshon and Blakemore, 1973; Greenlee et al., 1988), the bandwidth of orientational selectivity appears to be fairly independent of spatial frequency. The full bandwidth of the central 


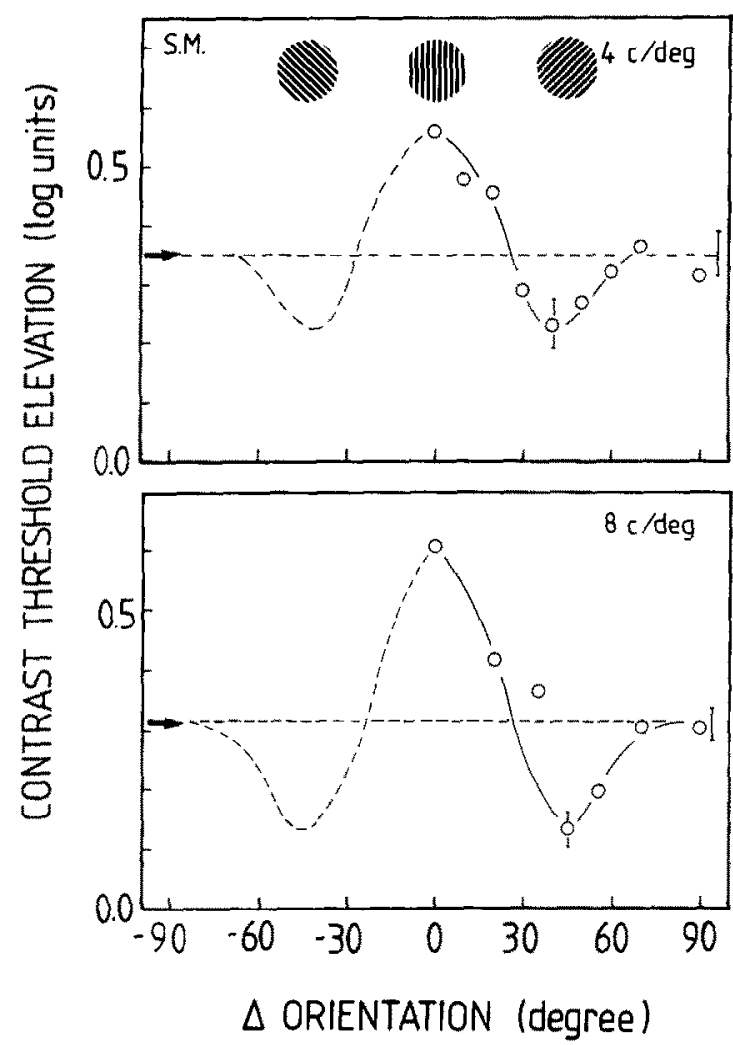

Fig. 4. The elevation in contrast threshold following adaptation to a $4 \mathrm{c} / \mathrm{deg}$ (upper panel) or a $8 \mathrm{c} / \mathrm{deg}$ (lower panel) vertical grating alternated with a grating of the same spatial frequency but of a different orientation as a function of the orientation difference between the first and second adapting gratings. The grating insets show the orientation of the second grating when it was identical to the first (centre) or when it had its most pronounced subtractive effect on adaptation (left and right). Data from observer SM.

adapting range is $45 \mathrm{deg}$, which corresponds well to that reported in single grating adaptation experiments (Blakemore and Nachmias, 1971; Movshon and Blakemore, 1973).

\section{DISCUSSION}

The technique of sequential adaptation enables us to isolate the neural interactions arising from adaptation to stimuli containing two spatial frequencies or orientations from the confounding effects of the luminance profile of complex gratings. Since the two adapting gratings were never simultaneously exposed in our experiments, any change in the resultant threshold elevation at the test frequency/ orientation must reflect the effect of the second adapting frequency/orientation acting on the mechanism processing the test grating. The results of the present experiments show that the amount and direction of change in threshold elevation induced by the second grating depend upon the relative difference in spatial frequency and orientation between the two adapting gratings. In both the spatial frequency and orientation domains sombrero-shaped adaptation profiles suggest that both facilitatory and inhibitory interactions occur.

Presumably, when the effects of the two adapting gratings sum they are both within the stimulus range responsible for adaptation in the mechanism tuned to the spatial frequency and orientation of the test grating. The width of this summative region is thus comparable to the spread of adaptation effects found in singlegrating adaptation experiments. Such comparisons indicate good agreement between the width of the summation region and the stimulus selectivity estimated by the conventional adaptation experiments for both spatial frequency (e.g. Blakemore and Campbell, 1969; Björklund and Magnussen, 1981; Georgeson and Harris, 1984) and orientation (e.g. Blakemore and Nachmias, 1971; Movshon and Blakemore, 1973).

The second subtractive region (below the dashed lines in Figs 2 and 4) would not be readily observed in single-grating adaptation because of the obvious "floor" effect set by the unadapted contrast thresholds. In our dualgrating experiments, the baseline is raised by 0.3 $\log$ units, thus allowing modulation of the contrast threshold both above and below this level. The fixed adapting grating thus is analogous to the conditioning stimuli which raise the base rate activity in single cells measured in neurophysiological experiments (e.g. Blakemore and Tobin, 1972; Nelson and Frost, 1978; Heggelund, 1986). There have, however, been some reports of threshold facilitation in singlegrating adaptation experiments (DeValois, 1977; Tolhurst and Barfield, 1978; Kelly and Burbeck, 1980). Furthermore, the illusory gratings which, after adaptation, briefly occur in blank fields, having apparent spatial frequencies and orientations remote from those of the adapting grating (Georgeson, 1976, 1980; Tyler and Nakayama, 1980), are probably manifestations of the same mechanism that yields subtractive effects in the present experiments (Georgeson, 1985).

Studies of suprathreshold aftereffects, which involve changes in the perceived size or orientation of stimuli also provide a non-zero baseline. Experiments on the tilt aftereffect have demonstrated interactions between adapting and test stimuli across the complete range of 90 deg difference in orientation, with negative 
(repulsion) aftereffects occurring up to 45-60 deg adapting angles and positive (attraction) aftereffects beyond (e.g. Campbell and Maffei, 1971). In addition, there is substantial evidence that similar inhibitory/disinhibitory processes are involved in the tilt aftereffect (Magnussen and Kurtenbach, 1980; Kurtenbach and Magnussen, 1981).

The subtractive sidebands evident in the results shown in Figs 2 and 4 provide a decisive test of two competing theories of spatial adaptation, namely the fatigue vs inhibition theory of adaptation. If threshold elevation were the result of neural fatigue (Swift and Smith, 1982; Georgeson and Harris, 1984), then one would not expect additional adaptation at more remote spatial frequencies or orientations to cause less adaptation. However, if one assumes that adaptation is an active process, which adjusts the contrast gain of the adapted mechanism (Ohzawa et al., 1985; Greenlee and Heitger, 1988) and that this gain adjustment may be mediated, at least in part, by lateral inhibition from mechanisms selective to neighbouring spatial frequencies and orientations (Blakemore et al., 1973; Dealy and Tolhurst, 1974), then interpretation of the results becomes simple. The central summative adaptation region in Figs 2 and 4 would be the result of inhibitory activity of neighbouring channels and the subtractive regions would be the results of these neighbours themselves becoming adapted and thus disinhibiting the mechanism tuned to the central frequency/orientation. The present results thus provide additional evidence for the inhibition theory of adaptation, which has been shown to account for a number of results concerning the tilt aftereffect (Kurtenbach and Magnussen, 1981; Magnussen and Johnsen. 1986). Objective evidence, which is based on visual evoked potential amplitude in human observers, for such inter-channel interactions in the orientation domain has been recently reported by Burr and Morrone (1987).

It is interesting to note here that, compared to the effect caused by an adapting grating interleaved with a blank, Klein and Stromeyer (1980, Fig. 3) found no difference in threshold elevation when a $f / 3$ adapting grating was interleaved with a $f$ adaptor using a $f$ test grating. There are, however, some important differences between the stimulus conditions used in their and our present experiments. First, the test and first adaptor, had a spatial frequency of $7.5 \mathrm{c} / \mathrm{deg}$, almost an octave above our $4 \mathrm{c} / \mathrm{deg}$ standard test grating. Second, as Klein and Stromeyer (1980) were interested in the interactions between harmonically related frequencies (of a squarewave) they used adapting contrasts that differed by a factor of three, $63 \%$ for $2.5 \mathrm{c} / \mathrm{deg}$ and $21 \%$ for $7.5 \mathrm{c} / \mathrm{deg}$, whereas we used an adapting contrast of $70 \%$ for both fixed and variable adapting gratings. The adaptation effect in their experiment was fairly small (about $0.2 \mathrm{log}$ unit) owning to the low adapting contrast at the $7.5 \mathrm{c} / \mathrm{deg}$ frequency. Furthermore, their mean luminance was $5 \mathrm{~cd} / \mathrm{m}^{2}$ compared to our $150 \mathrm{~cd} / \mathrm{m}^{2}$. The absence of an effect on threshold elevations in their experiment may thus be due to any or all of these differences.

In conclusion, the results from our dualgrating experiments provide evidence that lateral inhibition in the human visual cortex is organized along the dimensions of orientation and size (spatial frequency). Each neural mechanism receives excitatory input over a bandlimited range of spatial frequency and orientation and an inhibitory input beyond this range. Spatial adaptation experiments reveal the extent of this lateral inhibition. It is interesting to note here that such inhibitory interactions between neighbouring neurons plays an important role in recent models of the computation performed by neural networks (see Hopfield and Tank, 1986). There is increasing neurophysiological evidence for this type of inhibition in the visual cortex (Morrone et al., 1982; De Valois and Tootell, 1983; Nelson, 1985; Romoa et al., 1986), which might have its structural basis in an orderly organization of orientation columns and spatial frequency rows (Maffei and Fiorentini, 1977; Tootell et al., 1981; Berardi et al., 1982).

Acknowledgements--This research was supported by the Deutsche Forschungsgemeinschaft (SFB 325, B4). We thank L. Spillmann for providing laboratory facilities and Friedrich Heitger and Wilfred Ditterle for serving as subjects.

\section{REFERENCES}

Bernardi N., Bisti S., Cattaneo A., Fiorentini A. and Maffei L. (1982) Correlation between preferred orientation and spatial frequency of neurones in visual areas 17 and 18 of the cat. J. Physiol., Lond. 323, 606618.

Björklund R. A. and Magnussen S. (1981) A study of interocular transfer of spatial adaptation. Perception 10, 511-518.

Blakemore C. and Campbell F. W. (1969) On the existence of neurones in the human visual system selectively sensitive to the orientation and size of retinal images. J. Physiol., Lond. 203, 237-260. 
Blakemore C. and Nachmias J. (1971) The orientation specificity of two visual after-effects. J. Physiol., Lond. 213, 157-174.

Blakemore C. and Tobin E. A. (1972) Lateral inhibition between orientation detectors in the cat's visual cortex. Expl Brain Res. 15, 439 440.

Blakemore C., Muncey J. P. J. and Ridley R. M. (1973) Stimulus-specificity in the human visual system. Vision Res. 13, 1915-1931.

Braddick O., Campbell F. W. and Atkinson J. (1978) Channels in vision. Basic aspects. In Handbook of Sensory Physiology, Perception (Edited by Held R. Leibowitz H. W. and Teuber H.-L, ) pp. 3-38. Springer, Berlin.

Burr D. C. and Morrone M. C. (1987) Inhibitory interactions in the human vision system revealed by patternevoked potentials. J. Physiol., Lond. 389, 1-21.

Campbell F. W. and Maffei L. (1971) The tilt aftereffect: a fresh look. Vision Res. 11, 833-840.

Carpenter R. H. S. and Blakemore C. (1973) Interaction between orientations in human vision. Expl Brain Res. 18, 287.303.

Dealy R. S. and Tolhurst D. J. (1974) Is spatial adaptation an aftereffect of prolonged inhibition? J. Physiol., Lond. 241, 26 i -270.

De Valois K. K. (1977) Spatial frequency adaptation can enhance contrast scnsitivity. Vision Res. 17, 1057-1065.

De Valois K. K. and Tootell R. B. (1983) Spatial frequencyspecific inhibition in cat striate cortex cells. $J$. Physiol, Lond. 336, 359-376.

Georgeson M. A. (1976) Psychophysical hallucinations of orientation and spatial frequency. Perception 5, 99-111.

Georgeson M. A. (1980) The perceived spatial frequency, contrast and orientation of illusory gratings. Perception $\mathbf{9}$, 695-712.

Georgeson M. A. (1985) Inferring cortical organization from subjective visual patterns. In Models of the Visual Cortex (Edited by Rose D. and Dobson V. G.), pp. 223 232. Wiley, New York.

Georgeson M. A. and Harris M. G. (1984) Spatial selectivity of contrast adaptation: models and data. Vision Res. 24, 729.741

Greenlee M. W. and Heitger F. (1988) The functional role of contrast adaptation. Vision Res. 28, 791-797.

Greenlee M. W. and Magnussen S. (1987) Higher-harmonic adaptation and the detection of squarewave gratings. Vision Res. 27, 249-255.

Greenlee M. W. and Magnussen S. (1988) Spatial waveform discrimination following higher harmonic adaptation. J. op. Soc, Am. (In press).

Greenlee M. W. Magnussen S. and Nordby K. (1988) Spatial vision of the achromat: spatial frequency and orientation specific adaptation. J. Physiol., Lond. 395, 661678

Heggelund P. (1986) Quantitative studies of enhancement and suppression zones in the receptive field of simple cells in cat striate cortex. I. Physiol. Lond. 373, 293-310.

von der Heydt R. (1987) Approaches to visual cortical function. Rev. Physiol. Biochem. Pharmac. 108, 69-150.

von der Heydt R., Petcrhans E. and Dürsteler M. R. (1986) Spatial periodicity detection in monkey visual cortex. Neurosci. Abstr. 12, 1499.

Hopfield J. J. and Tank D. W. (1986) Computing with neural circuits: a model. Science, N.Y. 233, 625-633.

Kelly D. H. and Burbeck C. A. (1980) Motion and vision III. Stabilized pattern adaptation. J. opt. Soc. Am. 70 , 749. 754 .
Klein S. and Stromeyer C. F. III (1980) On inhibition between spatial frequency channels: adaptation to complex gratings. Vision Res. 20, 459-466.

Klein S., Stromeyer C. F. III and Ganz L. (1974) The simultaneous spatial frequency shift: a dissociation between the detection and perception of gratings. Vision Res. 14, 1421-1432.

Kurtenbach W. and Magnussen S. (1981) Inhibition, disinhibition and summation among orientation detectors in human vision. Expl Brain Res. 43, 193-198.

Lieberman H. R. and Pentland A. P. (1982) Microcomputer-based estimation of psychophysical thresholds: the best PEST. Behav. Res. Meth. Instrum. 14, 21-25.

Maffei L. and Fiorentini A. (1977) Spatial frequency rows in the striate visual cortex. Vision Res. 17, 257-264.

Magnussen S. and Greenlee M. W. (1985) Marathon adaptation to spatial contrast: saturation in sight. Vision Res. 25, $1409-1411$.

Magnussen S. and Greenlee M. W. (1986) Contrast threshold elevation following continuous and interrupted adaptation. Vision Res. 26, 673-675.

Magnussen S. and Johnsen T. (1986) Temporal aspects of spatial adaptation. A study of the tilt aftereffect Vision Res. 26, 661-671.

Magnussen S. and Kurtenbach W. (1980) Adapting to two orientations: disinhibition in a visual aftereffect. Science, N.Y. 207, 908-909.

Morrone M. C., Burr D. C. and Maffei L. (1982) Functional implications of cross-orientation inhibition of cortical visual cells. I. Neurophysiological evidence. Proc. R. Soc. $B$ 216, 335-354.

Movshon J. A. and Blakemore C. (1973) Orientation specificity and spatial selectivity in human vision. Perception 2, 53-60.

Nachmias J., Sansbury R., Vassilev A. and Weber A. (1973) Adaptation to square-wave gratings: in search of the elusive third harmonic. Vision Res. 13, 1335-1342.

Nelson J. A. (1985) The cellular basis of perception. In Models of the Visual Coriex (Edited by Rose D. and Dobson V. G.). Wiley. New York.

Nelson J. I. and Frost B. J. (1978) Orientation selective inhibition from beyond the classical visual receptive field. Brain Res. 139, 359-365.

Ohzawa I., Sclar G. and Freeman R. D. (1985) Contrast gain control in the cat"s visual system. $J$. Neurophysiol. $\mathbf{5 4}$, $651-667$.

Romoa A. S., Shadlen M., Skottun B. C. and Freeman R. D. (1986) A comparison of inhibition in orientation and spatial frequency selectivity or cat visual cortex. Nature, Lond. 321, 237-239.

Rose D. and Dobson V. G. (Eds) (1985) Models of the Visual Cortex. Wiley, New York.

Shapley R. and Lennie P. (1985) Spatial frequency analysis in the visual system. A. Rev. Neurosci. 8, 547-583.

Stecher S., Sigel C. and Lange R. V. (1973) Composite adaptation and spatial frequency interactions. Vision Res. 13, 2527-2531.

Swift D. J. and Smith R. A. (1982) An action spectrum for spatial-frequency adaptation. Vision Res. 22, 235-246.

Swift D. J. and Smith R. A. (1985) Adaptation to composite gratings: interaction or artifact? J. opt. soc. Am. (Suppl.) 2A, P80.

Tolhurst D. J, (1972) Adaptation to square-wave gratings: inhibition between spatial frequency channels in the human visual system. J. Physiol. Lond. 226, 231-248. 
Tolhurst D. I. and Barfield L. P. (1978) Interactions between spatial frequency channels. Vision Res. 18, 951.958.

Tootell R. B. H., Silverman M. S, and DeValois R. L. (1981)
Spatial frequency columns in primary visual cortex. Science, N.Y. 214, 813-815.

Tyler C. W. and Nakayama K. (1980) Grating induction: a new type of aftereffect. Vision Res. 20, 437-441. 